ORIENTAL JOURNAL OF CHEMISTRY

An International Open Free Access, Peer Reviewed Research Journal

www.orientjchem.org
ISSN: 0970-020 X

CODEN: OJCHEG

2017, Vol. 33, No.(5):

Pg. 2668-2672

\title{
A Comparison Between Different Properties of Bromoxynil and its Derivatives by DFT Method
}

\author{
R.P. SINGH GREWAL ${ }^{1 *}$ and GIRIJA SHARMA ${ }^{2}$ \\ ${ }^{1}$ Applied Sciences Department, Guru Nanak Dev Engineering College, Ludhiana. \\ ${ }^{2}$ Research Scholar IKGPTU, Jalandhar. \\ *Corresponding author E-mail: rpsinghgrewal@gmail.com \\ http://dx.doi.org/10.13005/ojc/330565
}

(Received: February 02, 2017; Accepted: April 05, 2017)

\begin{abstract}
In this work, we perform a set of quantum mechanical calculations on compounds bromoxynil and its derivatives. Various properties entropy, dipole moment, energy, IR spectra studied by using DFT method in Gaussian calculations. The solubility and inhibition power of these compounds compared with each other. IR frequencies compared with experimental values.
\end{abstract}

Keywords: DFT, Bromoxynil, Dipole moment, entropy, IR spectra.

\section{INTRODUCTION}

Agrochemicals have become a fundamental input in agriculture crop production and leading the industry to achieve its transformation in-line with the changing demands from the growers as well as to suit the changing weather patterns. There are many types of agrochemicals that are used to increase crop production. Basically agrochemicals are divided into two categories Fertilizers and Pesticides. Pesticides are instrumental in healthy rearing of crops, and are grouped according to the types of pests which they kill/inhibit/restrict. Various Pesticides are :

- Insecticides - Used to kill insects.

- $\quad$ Herbicides - Used to kill unwanted plants while leaving the desired crop relatively unharmed.
- Rodenticides - Used to kill rodents like squirrels, mice, chipmunks etc.

- $\quad$ Bactericides - Used to restrict and kill the bacteria.

- $\quad$ Fungicides- Used to control fungal diseases by inhibiting or killing the fungus. - $\quad$ Larvicides- Used typically against the larva life stage of the insect.

Bromoxynil is a nitrile herbicide used for post emergent control of annual broad leaves. Bromoxynil inhibit the process of photosynthesis of unwanted plants. Bromoxynil inhibits the electron transfer process of photosystem $\mathrm{II}^{1}$. Half life of bromoxynil in soil is few days. It is observed that bromoxynil is safe to use for broad leaf control in wheat during spring fall season ${ }^{2}$. Theoretic study of Bromoxynil and its derivatives has been carried out. The derivatives taken are 3, 5-Dibromo-4- 
methoxy benzoic acid (D-1), 3, 5-Dibromo-4-hydroxy benzoic acid (D-2), 2,6- dibromophenol (D-3), 3-bromo-4-hydroxy benzoic acid (D-4). Gaussian is used to study various properties. The method opted is DFT. With this method dipole moment, entropy, total energy, enthalpy, vibrational frequencies, and IR spectra has been studied.

Dipole moment decides the reactive nature of the herbicide and retention time of the agrochemical. More the dipole moment more will be the polarity and more will the reactivity of the herbicide ${ }^{3-4}$. Spontaneous processes are accompanied by increase in entropy as well as increase in the disorder of the system. A little consideration will show that when a spontaneous process takes place it moves from less probable state to a more probable state. Hence, there is a close relationship between entropy $S$ and the thermodynamic probability $\mathrm{W}$ of the state of the system. IR spectroscopy is used to study the residues of agrochemicals in food materials ${ }^{5}$. By DFT method various studies have been done. DFT is the most reliable method for theoretical study. The reliability has been studied for number of chemicals. The experimental values have good correlation with calculated values ${ }^{6-8}$.

A comparison between entropies of five aromatic compounds has been studied over a range of temperature ${ }^{9}$. These results have been compared with experimental results. The difference between the values by quantum mechanical method and statistical method is minor. So quantum and statistical calculations can be used simultaneously for investigation. Theoretical study of bis(N-(5-(4-methoxyphenyl)-1,3,4-oxadiazol-2$\mathrm{yl}$ )ethanimidamidato) $\mathrm{M}$ complexes ( $\mathrm{M}=\mathrm{Co}, \mathrm{Ni}, \mathrm{Cu}$, $\mathrm{Zn}, \mathrm{Pd}, \mathrm{Cd}$ ) have been carried out for their structural electronic and optical properties ${ }^{10}$. Theoretical results are in good agreement with experimental data. Study of 4-(2,3-Dihydroxy benzylideneaminol)3-methyl-1H-1.2.4-triazol-5(4H)-one has been done by DFT computational modeling ${ }^{11}$.

\section{Computational Methods}

We first perform the calculations to generate the structure of minimum energy using energy optimization of the compound. Energy optimization is done to get the geometrically most stable form of the compound. This calculation is done for frequency and energy optimization. The method used is DFT with basis set 3-21G. With this method and basis set calculations have been done for all the five compounds bromoxynil and its four derivatives i.e. D-1, D-2, D-3 and D-4. From these calculations we infer the dipole moment, entropy, polarizability, energy, vibrational frequencies and IR spectra for all the five chemicals. Here the molecular structures of these five chemicals have been used as an input in Gauss view 3.09 which is based on Gaussian. The IUPAC name, structure, and molecular weight of these compounds is given in Table 1.

Table 1

\begin{tabular}{ll}
\hline IUPAC Name of the Compounds & Molecular Weight \\
\hline 3,5-Dibromo-4-hydroxy benzonitrile(Bromoxynil) & $274.85814 \mathrm{amu}$ \\
3,5-Dibromo-4-methoxy benzoic acid (D-1) & $307.86837 \mathrm{amu}$ \\
3,5-Dibromo-4-hydroxy benzoic acid (D-2) & $293.85272 \mathrm{amu}$ \\
3,6-Dibromo phenol (D-3) & $249.86289 \mathrm{amu}$ \\
\hline
\end{tabular}




\section{RESULTS AND DISCUSSION}

\section{Dipole moment}

Dipole moment has been calculated for all the five compounds. The values of dipole moment have been compared with each other. The calculated values for dipole moment are given in table 2. Dipole moment explains the retention time of the agrochemical ${ }^{12}$. The more the dipole moment more will be the solubility of herbicide in water. Hence the chemical with greatest value of dipole moment will be removed easily and its contamination in soil and food materials will be less. As per results mentioned in table Bromoxynil will have minimum contamination in soil and food materials as compare to its derivatives. The chemicals will follow the following order contamination D-2 > D-4 > D-3 > D-1 > Bromoxynil.

Bromoxynil kills the unwanted plants, weeds by inhibiting the process of their food production that is the process of photosynthesis. Bromoxynil inhibits photosynthesis by binding to the Photosystem II complex of chloroplast membranes and blocking electron transport ${ }^{13}$. The efficiency of photosynthesis has been observed by Carnot cycle. In photosynthesis both photosystem I and II function with negative entropy production ${ }^{14}$. Entropy of herbicide affects the process of photosynthesis. As negative entropy enhances the process of photosynthesis so greater value of entropy may decrease the rate of reaction of photosynthesis. Entropy calculated for bromoxynil and its four derivatives is mentioned in table 2.

In table 2 derivative 1 has maximum entropy so it will act as strongest inhibitor in photosynthesis of weeds. The chemicals will follow the following order of inhibition power D-1 > D-2 > Bromoxynil > D-4 > D-3. Derivative 3 has minimum entropy so it will act as weakest herbicide. Total thermal energy produced by the herbicides is given in table 2. More the thermal energy more will be the effect of the herbicide. This heat will help to kill the weeds. The chemicals will follow the following order for destruction of weeds $D-1>D-4>D-2$ $>$ Bromoxynil>D-3 with respect to their thermal energies. Hence D-1 is the most effective herbicide. Thermal energy of D-3 is minimum therefore heat released by $D-3$ is minimum so its action towards killing of weeds will be weakest.

\section{IR spectra}

IR spectra of all the chemicals have been taken. IR spectroscopy is the analysis of interaction of infra red radiations with the molecule. The chemists used it to find out the functional groups present in a chemical or to find out the unknown compound. By use of IR spectra the contamination of herbicides in food materials, soil and water resources can be find out. The toxicity of the herbicides can be studied by using IR spectroscopy ${ }^{15-17}$. By the use of finger print region food adulteration can be detected. Food adulteration has been studied in China using finger print region ${ }^{18}$. These days IR spectroscopy is used to detect the diseases in the plants ${ }^{19}$. IR spectra of bromoxynil is taken as

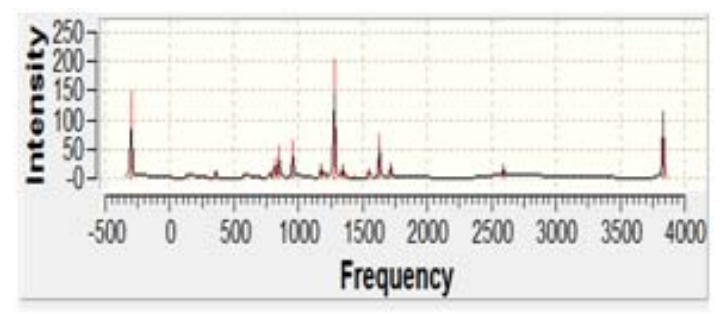

IR Spectra of Bromoxynil

Here one peak is observed near $3700 \mathrm{~cm}^{-1}$ which is the experimental frequency value for $\mathrm{O}-\mathrm{H}$ (stretch, free). Sharp peak has been observed for $\mathrm{C}-\mathrm{H}$ bending with value $1350-1480 \mathrm{~cm}^{-1}$ which is completely matches with experimental values. Multiple medium peaks have been observed between 1400-1600 which are for $C=C$.

Table 2

\begin{tabular}{lccccc}
\hline Name of the Compounds & Bromoxynil & D-1 & D-2 & D-3 & D-4 \\
\hline Dipole Moment (Debye) & 4.1082 & 2.0641 & 0.3434 & 1.8599 & 1.6561 \\
Entropy(Cal/Mol-Kelvin) & 101.032 & 116.127 & 106.395 & 92.027 & 97.701 \\
Total Thermal Energy (Kcal/Mol) & 58.468 & 87.145 & 68.733 & 58.186 & 73.834 \\
\hline
\end{tabular}


IR spectra for derivative 1 is taken as

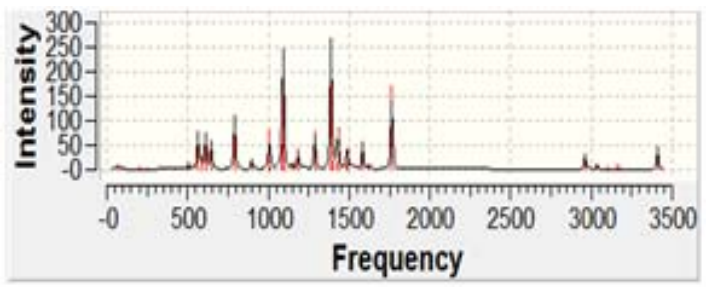

IR Spectra of D1

A strong peak has been observed near $1700 \mathrm{~cm}^{-1}$ which is lies in the frequency range 1700 $1725 \mathrm{~cm}^{-1}$ of carbonyl group. Multiple peaks have been observed for C-Br between $690-515 \mathrm{~cm}^{-1}$. Small peak has been observed at $2900 \mathrm{~cm}^{-1}$ for methoxy group. All these results fully agree with the experimental values.

IR spectra for D-2 is taken as

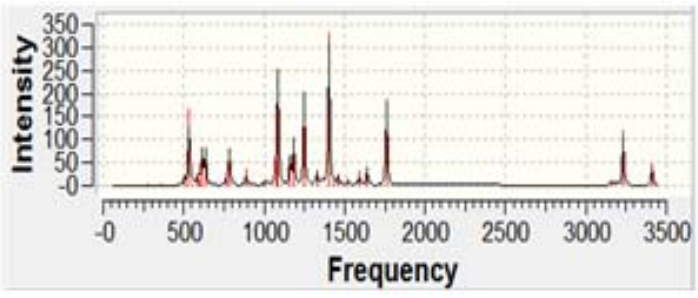

IR Spectra of D2

Sharp peak for $\mathrm{OH}$ group has been observed at $3300 \mathrm{~cm}^{-1}$. Experimental value for this group is range between $3200-3600$. The range for the $\mathrm{C}=\mathrm{O}$ group in an acid is $1700-1725 \mathrm{~cm}^{-1}$. Here we observed a sharp peak in this range. So it gives confirmation of $\mathrm{COOH}$ group. $\mathrm{C}-\mathrm{Br}$ give spectra in range $690-515 \mathrm{~cm}^{-1}$ here we observe the same as experimental values.

IR spectra for D-3 is taken as

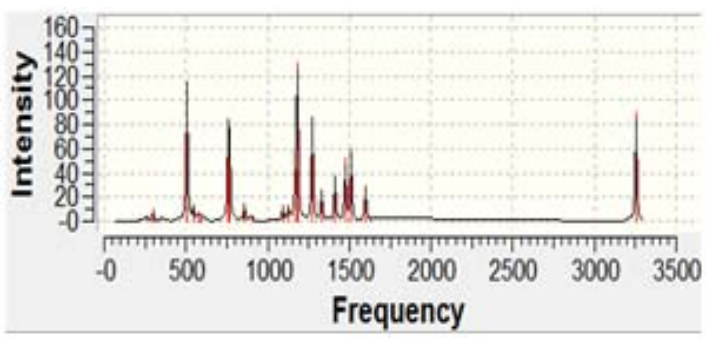

IR Spectra of D3

A sharp peak is observed at $3250 \mathrm{~cm}^{-1}$ which is lies in the range for O-H group (3200-3600) $\mathrm{cm}^{-1}$. Sharp peaks have been observed for $\mathrm{C}-\mathrm{Br}$ in range $690-515 \mathrm{~cm}^{-1}$. These values completely match with experimental values of functional groups.

IR spectra for D-4 is taken as

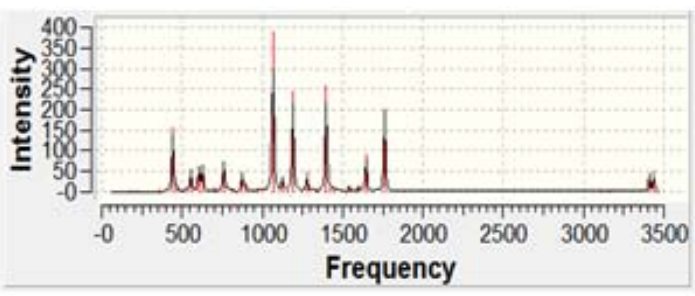

IR Spectra of D4

Peak for O-H group has been observed at $3400 \mathrm{~cm}^{-1}$. Experimental range for $\mathrm{O}-\mathrm{H}$ group is (3200-3600). For C-Br peaks have been observed between $690-515 \mathrm{~cm}^{-1}$ as per experimental values. The range for the $\mathrm{C}=\mathrm{O}$ group in an acid is 1700 $1725 \mathrm{~cm}^{-1}$. Here we observed a sharp peak in the same range. So it gives confirmation of $\mathrm{COOH}$ group.

Herbicides Bromoxynil and its four derivatives are discussed. With the calculation of dipole moment bromoxynil has found with minimum retention time in soil and minimum contamination in soil and food materials. With Entropy and Total thermal energy calculation it is found that D-1 has maximum value of entropy and total thermal energy. Hence Derivative-1(3, 5-Dibromo-4-methoxy benzoic acid) is the most effective herbicide. Moreover chemical D-3(2, 6-Dibromo phenol) has minimum value of entropy and total thermal energy, so it is the least effective herbicide among all the chemicals taken. The values for vibrational frequency of all the functional groups in IR spectra of all the chemicals taken by Gaussian are compared with the experimental values of functional group gives the same result. So the IR spectra taken by Gaussian give the valid results. We can study the path of action of herbicide by taking IR spectra using DFT method.

\section{ACKNOWLEDGEMENT}

Authorsacknowledge the facilities provided by IKGPTU, Kapurthala and GNDEC, Jalandhar. 


\section{REFERENCES}

1. Sonderskov M.; Swanton C.J.; Kudsk P.; An International Journal of Weed Biology, Ecology and vegetation Management, 2012,52, 169-177.

2. Reddy S.S.; Stahlman P.W.; Geier P.W; Peterson D.E.; American Journal of Plant Sciences, 2012,3, 1613-1618.

3. Dwivedi A.; Pandey A.K.; Sonwani S.; Netam R.; Bajpai A.; Journal of Chemical and Pharmaceutical Research, 2014, 6(12), 411-417.

4. Bajpai A.; Pandey A.K.; Pandey K.; Dwivedi A.; Journal of Computational Methods in Molecular Design, 2014, 4(1) 64-69.

5. Teye E.; Huang X.; Afoakwa N.; American Journal of Food Science and Technology, 2013, 1(1), 1-8.

6. Bauza A.; Alkorta I.; Frontera A.; Elguero J.; Journal of Chemical Theory and Computation, 2013, 9 (11), 5201-5210.

7. Liu K.; Li X.Y.; Wang B.; J. Comput. Chem., 2014, 35 (9), 7703-710.

8. Khalili B.; Rimaz M.; Current Chemistry Letters, 2016, 5, 7-18.

9. Kassaee M.H.; Keffer D.J.; Steele W.V.; Journal of molecular Structure: Theochem,
2007, 802, 23-34.

10. Djebli Y.; Bencharif M.; Rabilloud F.; Computational and Theoretical Chemistry, 2016, 1080, 16-22.

11. Tanak H.; Computational and Theoretical Chemistry, 2011, 967, 93-101.

12. Plakas K.V.; Karabelas A.J.; Wintgens T.; Melin T.; Journal of Membrane Science, 2006, 284, 291-300.

13. Taghipour F.; Huyap F.; Journal Teknologi, 2012.

14. JenningsR.C.; Engelmann E.; Garlaschi F.; Casazza A.P.; Zucchelli G.; Elsevier, Biochimica et Biophysica Acta, 2005, 1709 , 251-255.

15. Dakhakhni T. H.; Raouf G.A.; Qusti S.Y.; European Biophysics Journal, 2016, 45 (4), 311-320.

16. Salmain M.; Durand N.F.; Pradier C.M.; Analytical Biochemistry, 2008, 373 (1), 61-70.

17. Srinivasan R.; Advances in Materials Science and Engineering, 2011, 2011, 1-17.

18. Zhang J.; Zhang X.; Dediu L.; Victor C.; Food Control, 2011, 22, 1126-1135.

19. Sankaran S.; Mishra A.; Ehsani R.; Davis C.; Computers and Electronics in Agriculture, 2010, 72, 1-13. 